\title{
Design and Calibration of a Polychromator for the Thomson Scattering at Wendelstein 7-X
}

\author{
S. Schmuck ${ }^{1}$, A. Dinklage ${ }^{1}$, R. Fischer ${ }^{2}$, J.P. Knauer ${ }^{1}$, B. Kurzan ${ }^{2}$, \\ H.-D. Murmann ${ }^{2}$, E. Pasch ${ }^{1}$ \\ Max - Planck - Institut für Plasmaphysik, EURATOM Association, \\ ${ }^{1}$ Wendelsteinstr.1, 17491 Greifswald, ${ }^{2}$ Boltzmannstr. 2, 85748 Garching, Germany
}

\begin{abstract}
On Wendelstein 7-X, electron density and electron temperature profiles are planned to be measured by Thomson scattering. The specific system employs fiber bundles for the scattered light which is detected by means of a five-channel polychromator system. The detection system is assembled and its components are characterized. A calibration technique employing a super-continuum light source is assessed by comparison with a calibration technique using an optical parametric oscillator laser system. The characterization is preparing further diagnostic optimization studies employing a virtual instrument.
\end{abstract}

Keywords: Thomson scattering diagnostic, polychromator, dichroitic mirror, super-continuum light source

\section{INTRODUCTION}

Thomson scattering (TS) is envisaged as a standard diagnostic for both electron temperature $\left(T_{e}\right)$ and electron density $\left(\mathrm{n}_{\mathrm{e}}\right)$ profile measurements on Wendelstein 7-X [1]. As an essential part of the diagnostic setup the spectral analysis of the scattered light is performed with polychromators for the so-called bulk Thomson scattering system which is expected to deliver full profiles of $n_{e}$ and $T_{e}$. The Wendelstein 7-X polychromator is based on the ASDEX Upgrade detection system [2] which will be fed by scattered light through an optical fiber bundle, see e.g. [3]. The design of the Thomson scattering system allows a couple of optimization steps. In order to assess the effect of design measures, studies by means of a virtual diagnostic accompany the hardware developments. This virtual diagnostic simulates the measured data from given physical quantities, i.e. $n_{e}$ and $T_{e}$. This allows one to quantify the impact of different diagnostic components. This assessment is the basis for detailed optimization studies which can be performed by an optimization of the expected information gain, for more details on this concept see [4,5]. Furthermore, the calibration of the spectral response of the polychromator is essential for determining the overall performance of the diagnostic and - particularly - the uncertainties of the measured quantities.

This paper describes the status of the hardware development of the W7-X TS polychromator. A calibration procedure employing a super-continuum light source is compared to calibration with an optical parametric oscillator (OPO).

\section{Design of the Polychromator}

Electron density and electron temperature profiles in W7-X are expected to cover $5 \cdot 10^{18} \mathrm{~m}^{-3}<\mathrm{n}_{\mathrm{e}}<4 \cdot 10^{20} \mathrm{~m}^{-3}$ and $20 \mathrm{eV}<\mathrm{T}_{\mathrm{e}}<10 \mathrm{keV}$ with some safety margins. In order to prepare a robust $\mathrm{n}_{\mathrm{e}}$ and $\mathrm{T}_{\mathrm{e}}$ diagnostic, a polychromator system was favoured instead of TV-TS [6,7] which will be used for edge studies.

At Wendelstein 7-X, the Thomson scattered spectrum from the examined scattering volume is guided to the polychromator by an optical fiber bundle with the numerical aperture of NA $=0.37$. Inside the polychromator the spectrum is dispersed into separate spectral channels. The polychromator design is shown in figure 1a. The main optical components along the optical path are the dichroitic mirror and 5 interference filters. Therefore, five spectral channels are available plus an additional channel collecting light which is transmitted by the dichroitic mirror. 


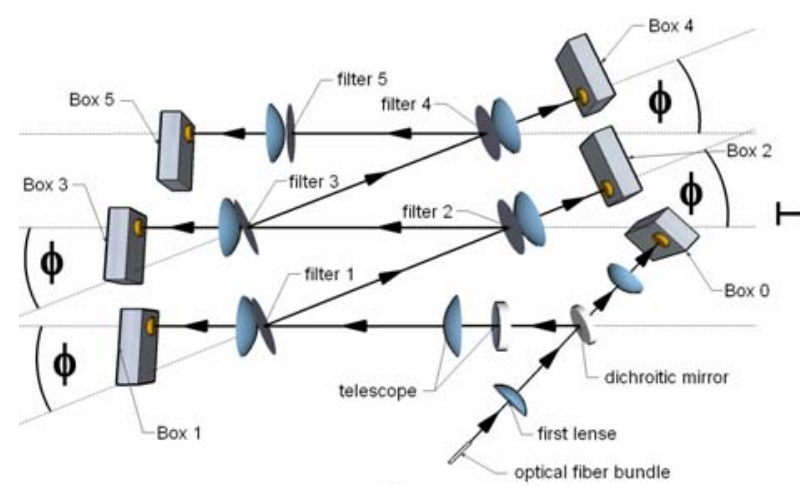

(a)

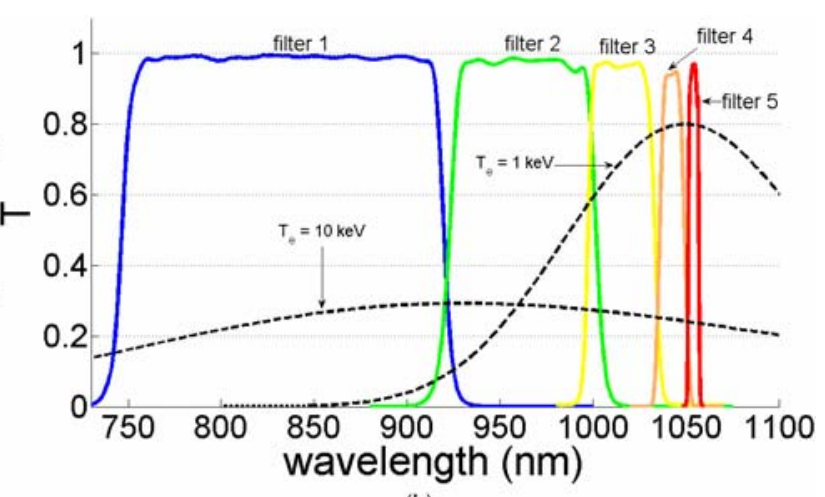

(b)

Figure 1: (a) Arrangement of the polychromator for Wendelstein 7-X. The angel $\phi$ is 22 degrees. (b) Transmission curves of the interference filters. Thomson scattering spectra are also shown for two electron temperatures $\left(T_{e}=1 \mathrm{keV}, \mathrm{T}_{e}=10 \mathrm{keV}\right)$ for the wavelength of a Nd-YAG laser (1064 nm).

Every spectral channel includes a converging lens and a detector. The used detectors are silicon avalanche photodiodes (Perkin Elmer, Type C30659-1060-3A) which are temperature stabilized at $29^{\circ} \mathrm{C}$ to achieve a constant amplification of the avalanche electrons. The transmission curves of the used interference filters (BARR associates, Inc.) and two calculated Thomson scattering spectra are shown in figure 1b. The filters cover the spectral range below the laser wavelength of $1064 \mathrm{~nm}$ in the range from $750 \mathrm{~nm}$ to $1058 \mathrm{~nm}$. The spectral positions and widths of the filters are chosen to guarantee a sufficient signal-to-noise ratio (SNR) for temperature measurements up to $10 \mathrm{keV}$. The filters have steep flanks and high transmission values.

For the Thomson scattering diagnostics very powerful lasers ( $\mathrm{P}=100 \mathrm{MW}, \mathrm{t}=10 \mathrm{~ns})$ are applied. When the laser light passes the windows of the plasma vessel additional photon scattering can occur. These photons can disturb the measurement if they arrive the avalanche diodes. This effect is an error source for the $n_{e}$ and $T_{e}$ measurement. To reduce the intensity of the laser stray light the filters damp the intensity at this wavelength by at least 4 orders of magnitudes. In addition, a dichroitic mirror is also integrated into the polychromator. For a characterization of the mirror, we measured the transmission for different polarization conditions of the incidenced light (s-, p- and non-polarized) and different angels of incidence (figure 2). The transmission maximum at $1064 \mathrm{~nm}$ can be adjusted to the laser wavelength by tilting the mirror. The scattered light is polarized and this has to be considered in the case of direct imaging to the polychromator. For p-polarized light at an angel of incidence of 20 degree, the dichroitic mirror shows at $1064 \mathrm{~nm}$ a transmission of $80 \%$ and the reflectivity is about $99 \%$ in the range from $850 \mathrm{~nm}$ up to $1020 \mathrm{~nm}$. In this range, the Thomson scattering spectrum is reflected nearly unaffected to the spectral channels. The so-called Raman filter (filter 5, 1051-1058 $\mathrm{nm}$ ) lies in the flank of the dichroitic mirror and the reflectivity varies from $85 \%$ at $1051 \mathrm{~nm}$ to $64 \%$ at $1058 \mathrm{~nm}$.

Since, the light from an optical fiber bundle is non-polarized the properties of the dichroitic mirror were examined with non-polarized light. The transmission for this polarisation state has a value of only $40 \%$ at $\lambda=1064 \mathrm{~nm}$. This is not sufficient for reduction of laser stray light. Therefore, the usage of the dichroitic mirror must be reconsidered for polychromators at Wendelstein 7-X but may have potential for other.

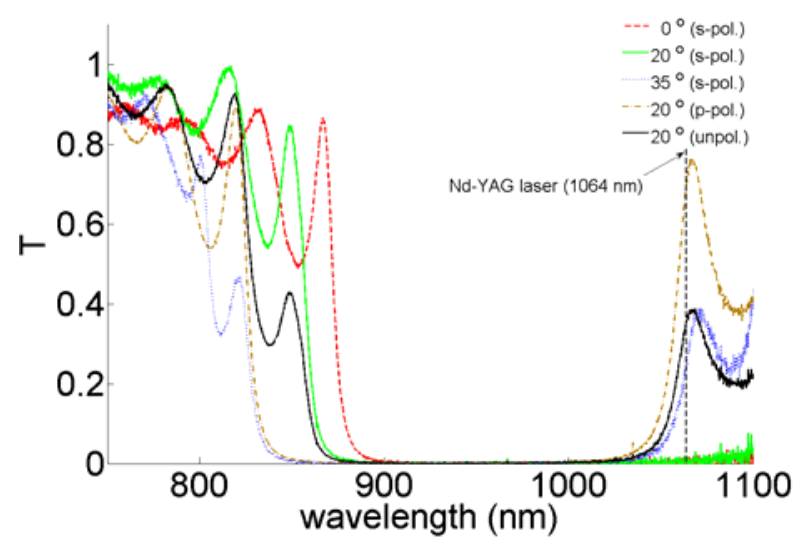

Figure 2: Transmission of the dichroitic mirror for incident s-, p- and non-polarized light. 


\section{Calibration Setup}

The measurement of the electron temperature and density requires spectral calibration of the polychromator, i.e. to measure the spectral response of all spectral channels. The pulse length of the calibration source was found to result in systematic, spectrally dependent errors, therefore the calibration has to be performed providing light pulses similar to the pulse of the Nd-YAG laser $(\Delta \mathrm{t}=10 \mathrm{~ns})$. An established setup uses an optical parametric oscillator ( $\Delta \mathrm{t}=9 \mathrm{~ns})$ which is a laser light source with tuneable laser wavelength. An alternative setup uses a super-continuum light source [4]. Our implemented setup (figure 3a) is adapted from [4]. The used supercontinuum light source (SLS) has the following features: The broadband spectrum covers the range from $500 \mathrm{~nm}$ to $2000 \mathrm{~nm}$. The power spectrum in the relevant band is shown in figure $3 \mathrm{~b}$. The source is pulsed with a frequency of $30 \mathrm{kHz}$ with pulse duration of $1-2 \mathrm{~ns}$. The mean output power per pulse is $120 \mathrm{~mW}$. The light output of the SLS is a flexible fiber with NA=0.23. The SLS is compact and easily transportable.

In our setup (Fig. 3a) the spectrum of the SLS passes a red filter. The light with wavelengths below $610 \mathrm{~nm}$ is reflected and illuminates a diode which triggers the measurement. Furthermore the red filter suppresses light of higher orders passing the grating monochromator. The quasi monochromatic light $(\Delta \lambda=0.5 \mathrm{~nm})$ leaves the monochromator and reaches the quartz beam splitter. The transmitted light illuminates the reference diode which serves as an intensity monitor. The reflected part from the beam splitter is coupled into the fiber bundle. This bundle guides the light into the polychromator. The signals of the spectral channels are normalized to the reference diode signal. The wavelength dependent spectral response of the polychromator spectral channels can be derived by scanning the spectral range from $750 \mathrm{~nm}$ to $1100 \mathrm{~nm}$.

The results of the calibration measurement for the SLS setup is shown figure 4a. The broad curves are regression polynominals to the raw data of the spectral response for every channel and the dichroitic mirror channel. The error bands show the $1-\sigma$ deviation of the data to the regression polynomial (dashed lines).

The results for this calibration method can be compared with a method which is established at the fusion device ASDEX Upgrade. At ASDEX Upgrade, the setup contains an optical parametric oscillator (OPO) which is pumped by a Nd-YAG pump laser at $\lambda=355 \mathrm{~nm}$. The wavelength of the OPO is tuneable and the relevant spectral range can be scanned $(\Delta \lambda=0.3 \mathrm{~nm})$ to obtain the spectral response of the polychromator spectral channels.

The results for both calibration setups are shown in figure $4 \mathrm{~b}$. The black lines mark the response measured with the OPO setup surrounded with the 1- $\sigma$ confidence interval (dashed black lines). The spectral response received with the SLS setup is given by the coloured curves (see figure 4a).

A comparison of the SLS and OPO setup shows that the plateau for channel 1 and 3 coincidence within the error bands. For channel 2, a significant step in the SLS data can be seen, which lies, however, within the error band of the OPO measurement. Deviations from the 1- $\sigma$ error band can be seen in channel 4 and 5 and for the dichroitic mirror. The reason for these discrepancies could be due to an error of the spectral calibration of the reference diode. The reference diode is a silicon photo diode and the spectral response decreases very strongly at this spectral range. Therefore, the discrepancy should be resolved, even though the Thomson signals might not be significantly affected, since this filter covers the Raman lines for density calibration.

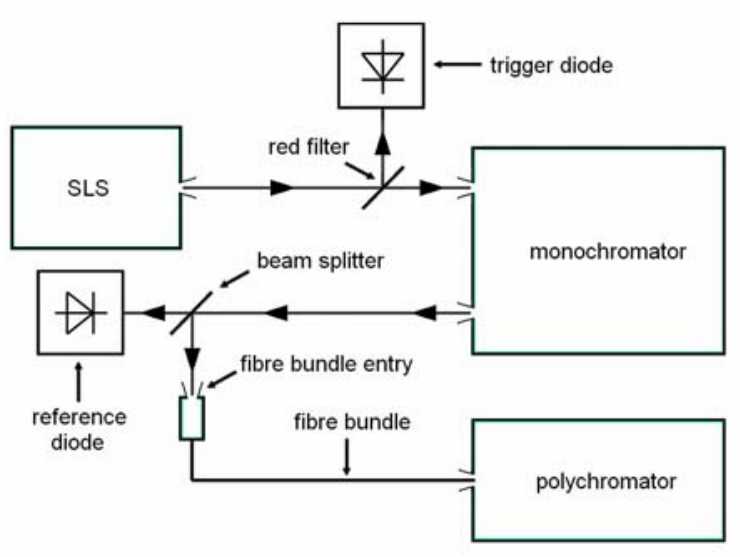

(a)

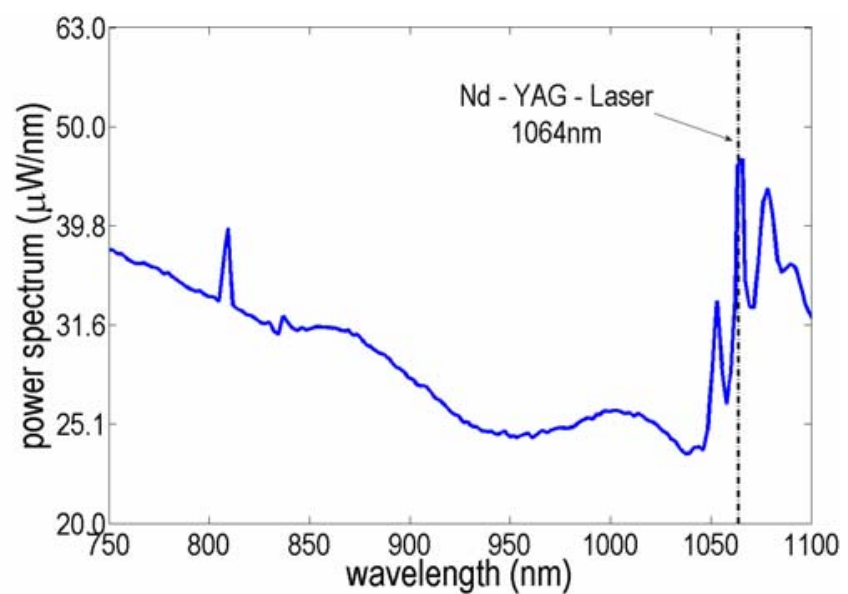

(b)

Figure 3: (a) Calibration setup. The spectrum of a super-continuum light source (SLS) passes a monochromator. The signals of the polychromator spectral channels are compared with the reference diode signals in the spectral range from 750nm to $1100 \mathrm{~nm}$. (b) Power spectrum of the super-continuum light source (SLS) [9]. The ordinate has a logarithmic scale. The pump laser wavelength at $1064 \mathrm{~nm}$ is marked by the dashed line. 

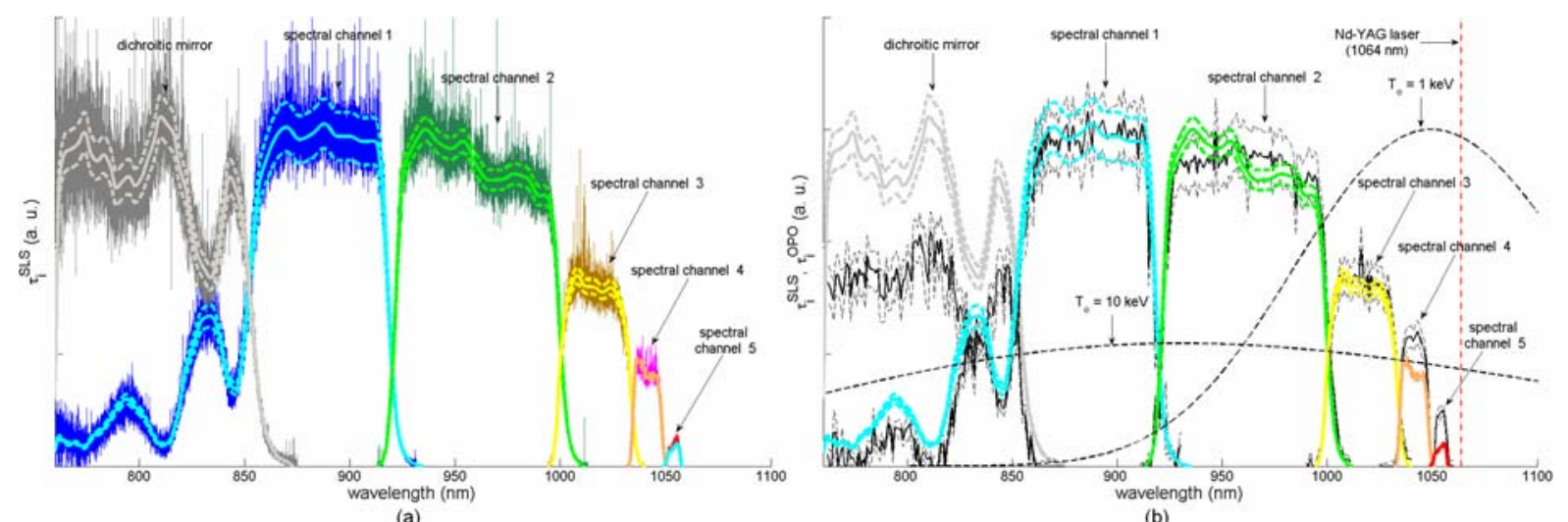

(a)

(b)

Figure 4: (a) Calibration results with the SLS setup for the W 7-X polychromator. The raw data of the spectral response ( $\tau_{\mathrm{i}}^{\text {SLS }}$ ) are the noisy graphs. A regression polynomial with the 1- $\sigma$ confidence interval is given by the lines and dashed lines. (b) Comparison of the spectral response measured with SLS setup ( $\tau_{\mathrm{i}}^{\text {SLS }}$, coloured) and with OPO setup ( $\tau_{\mathrm{i}}^{\text {OPO }}$, black). The error bands are given by the dashed lines. The Nd-YAG laser wavelength $(1064 \mathrm{~nm})$ is marked by the vertical line. Scattering spectras are shown for $\mathrm{T}_{\mathrm{e}}=1 \mathrm{keV}$ and $\mathrm{T}_{\mathrm{e}}=10 \mathrm{keV}$.

\section{SUMMARY}

A fiber coupled polychromator for the Thomson scattering on Wendelstein 7-X has been assembled and was successfully operated. Extending previous setups, the polychromator consists of five channels to provide high flexibility. The scattered light was detected with temperature stabilized avalanche diodes. The effect of a dichroitic mirror for suppression of stray light was examined. The investigations showed a lack of efficiency for non-polarized light occurring due to fiber bundle transmission. For polarized scattered light detection (direct imaging), however, the dichroitic mirror was shown to be an attractive option for the suppression of stray light. For intensity calibration, a technique employing a super-continuum light source (SLS) was compared to an OPO based technique. The results depend on the spectral range investigated: both consistency and discrepancy was found depending on the specific filter. It will be investigated if the spectral response of a reference detector in the SLS setup can be attributed to the discrepancies since the spectral response of the detector varies most in the regions of highest discrepancy.

In summary, the SLS method appears to be an attractive option to supplement the OPO calibration. Further studies of the effect of the discrepancies in the spectral calibrations on the analysis of $n_{e}$ and $T_{e}$ which use the calibration curves as an input for a virtual instrument are underway.

\section{ACKNOWLEDGMENTS}

The authors thank J. Hausmann and K. Lehmann for the technical support.

\section{REFERENCES}

1. H. J. Hartfuss et al, “Diagnostic strategy of the W7-X stellarator”, Rev. Sci. Instrum. 68, 1244 (1997).

2. H. Murmann, S. Götsch, H. Röhr, H. Salzmann and K. H. Steuer, "The Thomson scattering system of the ASDEX upgrade tokamak“, Rev. Sci. Instrum. 63, 4941 (1992).

3. T. N. Carlstrom, et al., Rev. Sci. Instrum. 61, 2858 (1990).

4. R. Fischer, "Bayesian Experimental Design -- Studies for Fusion Diagnostics“, "Bayesian Inference and Maximum Entropy Methods in Science and Engenieering“, AIP Conference Proceedings 76 (2004).

5. H. Dreier, A. Dinklage, R. Fischer, M. Hirsch and P. Kornejew, "Bayesian design of plasma diagnostics“, Rev. Sci. Instrum. 77, 10F323 (2016).

6. H. J. van der Meiden et al.,“10 kHz repetitive high-resolution TV Thomson scattering on TEXTOR: Design and performance (invited)”, Rev. Sci. Instrum. 77, 10E512 (2006).

7. J. P. Knauer, E. Pasch and G. Kühner, and the W7-AS Team, "High-resolution ruby laser Thomson scattering diagnostic for the W7-AS stellarator", Rev. Sci. Instrum. 74, 1679 (2003).

8. R. Pasqualotto and A. Alfier, "Thomson scattering calibration with ultrabright supercontinuum light source", Rev. Sci. Instrum. 77, 10E502 (2006).

9. KOHERAS A/S, Denmark, SuperK Test Report, Type: SCB-RED 100-PC 